\title{
eJRIEPS
}

Ejournal de la recherche sur l'intervention en éducation physique et sport

$27 \mid 2012$

Varia

\section{Les activités curriculaires des enseignants d'EPS, entre prescription et liberté : une revue de littérature}

Curricular activities of PE teachers, between prescription and freedom: a literature review

\section{Benoît Lenzen}

\section{OpenEdition}

Journals

Édition électronique

URL : http://journals.openedition.org/ejrieps/3103

ISSN : 2105-0821

\section{Éditeur}

ELLIADD

Référence électronique

Benoît Lenzen, "Les activités curriculaires des enseignants d'EPS, entre prescription et liberté : une revue de littérature », eJRIEPS [En ligne], 27 | 2012, mis en ligne le 01 juillet 2012, consulté le 20 novembre 2019. URL : http://journals.openedition.org/ejrieps/3103

Ce document a été généré automatiquement le 20 novembre 2019.

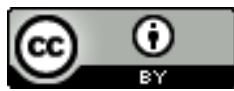

La revue eJRIEPS est mise à disposition selon les termes de la Creative Commons Attribution 4.0 International License. 


\title{
Les activités curriculaires des enseignants d'EPS, entre prescription et liberté : une revue de littérature
}

\author{
Curricular activities of PE teachers, between prescription and freedom: a \\ literature review
}

Benoît Lenzen

1 Comprendre la manière dont les enseignants d'EPS prennent leurs décisions en matière de choix des contenus à enseigner et les influences qui s'opèrent sur ces choix présente un intérêt majeur pour la recherche sur l'intervention en EPS, mais aussi pour la formation des enseignants d'EPS. Pour ce faire, il semble qu'il faille se doter d'un regard à la fois didactique et sociologique, en rupture avec le traditionnel cloisonnement des disciplines scientifiques (Johsua \& Lahire, 1999 ; Lahire, 2007). En effet, la perspective didactique permet d'éclairer le système enseignement-apprentissage, en tentant de répondre à des questions fondamentales telles que: qu'est-ce qui s'enseigne? De quelles manières? Quelles sont les relations entre pratiques scolaires et pratiques sociales? Mais l'étude des activités curriculaires de l'enseignant devrait aussi prendre en compte les «dispositions incorporées " par ce dernier (produites par l'expérience passée), les « contraintes contextuelles » qui pèsent sur lui et les marges de liberté dont il dispose. La perspective sociologique permet de mettre à jour ces paramètres de la situation qui se sont bien souvent joués avant ou ailleurs, faisant œuvre de dévoilement de ce qui demeure trop souvent implicite ou caché aux yeux du didacticien (Poggi, Verscheure, Musard \& Lenzen, 2010). C'est donc un double cadrage théorique qu'il convient d'élaborer pour investiguer le rôle de l'enseignant dans la construction des curricula d'EPS, articulant plusieurs théories, modèles et concepts respectivement issus des champs et sous-champs didactiques et sociologiques. 


\section{Un cadrage théorique socio-didactique}

\section{1. Les théories, modèles et concepts didactiques}

2 Initialement introduit par un sociologue (Verret, 1975) puis repris et développé par un didacticien des mathématiques (Chevallard, 1985) qui en a fait l'un des étendards de la didactique des disciplines, le concept de transposition didactique préfigurait le rapprochement auquel on assiste actuellement entre sociologie et didactique. Il désignait alors la transformation du savoir, à laquelle nul n'échappe lorsqu'il veut le transmettre à autrui. Ce concept étant largement décrit dans la littérature et dans certains articles publiés dans cette revue (e.g., Musard, Mahut \& Robin, 2002), nous ne le développerons pas pour lui-même ici, mais nous nous étendrons quelque peu sur les amendements qu'il a subis au fil du temps et au gré de ses emprunts par les autres didactiques des disciplines scolaires, avant de montrer en quoi la théorie ainsi élargie de la transposition didactique peut aider à la compréhension des activités curriculaires des enseignants d'EPS.

La théorie de la transposition didactique, initialement pensée pour rendre compte du passage du savoir savant au savoir à enseigner (transposition didactique externe) puis au savoir enseigné (transposition didactique interne), a très vite montré ses limites dès lors qu'il s'est agi de l'importer dans des disciplines scolaires qui n'ont pas, à l'inverse des mathématiques, des sciences naturelles et des sciences humaines et sociales, comme uniques ou principales références des savoirs savants. Par conséquent, certaines (remises en) questions ont émergé à son propos : le concept de transposition didactique n'est-il propre qu'aux mathématiques (Johsua, 1996) ? La théorie de la transposition didactique est-elle transposable (Caillot, 1996)? Pour dépasser ces limites, Johsua a proposé d'étendre la théorie de la transposition didactique aux savoirs experts. Empruntant une autre voie, Martinand (1981) avait proposé la notion de pratique de référence, permettant « de prendre en compte non seulement les savoirs en jeu, mais les objets, les instruments, les problèmes, les tâches, les contextes et rôles sociaux, d'où le terme de pratique, précisé et renforcé, sans doute avec redondance, en pratique sociale" (Martinand, 2001, p. 19). Synthétisant ces deux propositions, Perrenoud (1998) a considéré que l'on pouvait désormais travailler avec deux sources de la transposition didactique: d'une part des savoirs savants ou experts, d'autre part des pratiques sociales.

Toutefois, un autre élément est à considérer si l'on veut que la théorie de la transposition didactique puisse réellement rendre compte de la manière dont fonctionne le système d'enseignement-apprentissage actuel. Depuis une vingtaine d'années en effet, on est passé dans la plupart des pays occidentaux d'une pédagogie par objectifs, d'inspiration behavioriste, à une pédagogie par compétences, référée aux théories de l'expertise et de la cognition située (Crahay, 2006). L'idée d'une transposition de savoirs, fussent-ils experts, ne cadre plus avec la pédagogie par compétences, laquelle implique la mobilisation articulée de ressources dont les savoirs ne représentent qu'un élément-certes essentiel-parmi d'autres (savoir-faire, attitudes...). Aussi, Perrenoud (1998) a singulièrement complexifié le schéma de la transposition didactique (figure 1). Cette complexification de la chaîne de transposition porte d'abord sur la source même de la transposition. Contrairement aux savoirs savants immédiatement visibles, les pratiques nécessitent d'être décortiquées, explicitées avant de pouvoir être transmises. Les compétences au cœur de ces pratiques 
doivent être identifiées, et cela ne se fait pas sans heurts, désaccords et compromis. Une fois ces compétences identifiées, encore faut-il savoir de quoi elles sont faites, puis comment, à l'aide de quels dispositifs elles vont pouvoir être développées chez les apprenants. Ce travail complexe et exigeant, réalisé par la noosphère dans la théorie de Chevallard (1985), aboutit au "curriculum formel», susceptible de servir de base à l'enseignant pour élaborer le « curriculum réel ».

Figure 1. Transposition didactique à partir de pratiques (Perrenoud, 1998)

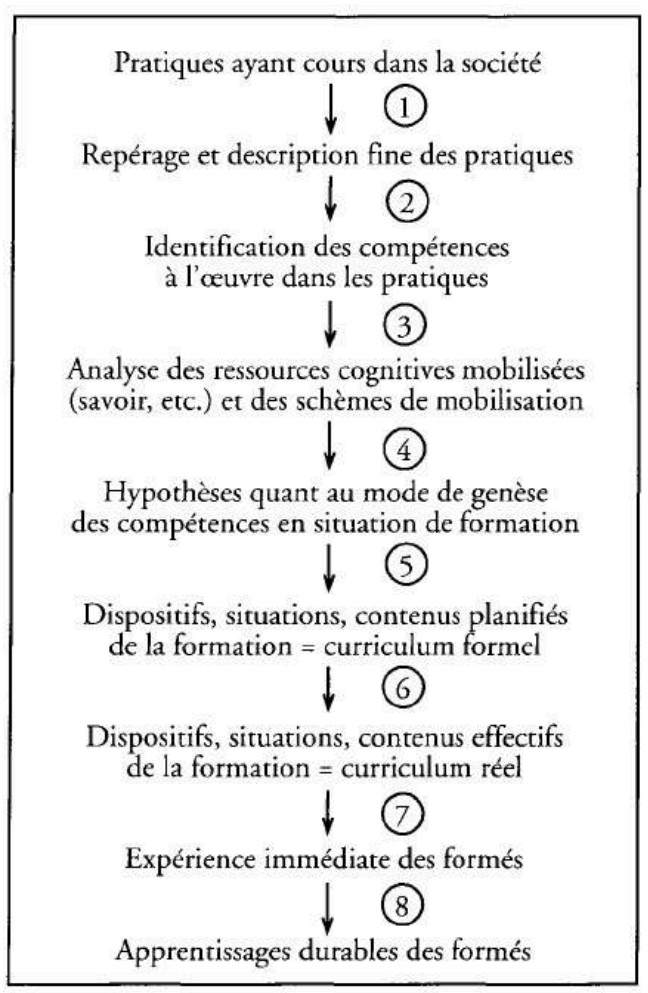

3 Ainsi amendée, la théorie de la transposition didactique offre un cadre de référence intéressant pour comprendre le cheminement des pratiques (incluant les savoirs y relatifs), depuis les institutions où elles sont apparues jusqu'à l'école. Ce cadre théorique permet de saisir quelles décisions les enseignants d'EPS prennent en matière de choix des contenus à enseigner, et l'écart qui en résulte entre les pratiques de référence et ce qu'ils proposent concrètement à leurs élèves. En revanche, il n'offre pas véritablement la possibilité d'éclairer le comment et le pourquoi de ces décisions. D'autant que ce type de modèle linéaire, sous une forme descendante, ne rend pas compte des boucles de rétroaction et autres influences - Martinand (2001) parle d'une "composition sous influences»-qui peuvent orienter l'activité curriculaire des enseignants. L'expérience des formés peut par exemple influencer le choix des compétences à enseigner. Plusieurs sous-champs de la sociologie peuvent par contre jouer ce rôle.

\section{2. Les théories, modèles et concepts sociologiques}

4 La sociologie du curriculum, née en Grande-Bretagne dans les années 1970 avant d'être diffusée par Forquin $(1983,2008)$ dans les pays francophones, s'est tout particulièrement intéressée aux conditions sociales de production des contenus du 
curriculum, lequel est défini comme "l'ensemble, institutionnellement prescrit et fonctionnellement différencié et structuré, de tout ce qui est censé être enseigné et appris, selon un ordre déterminé de programmation et de progression, dans le cadre d'un cycle d'études donné "(Forquin, 2008, p.8). Sous le regard des sociologues britanniques, la vision du curriculum est passée de celle d'une composante «naturelle » du monde scolaire à celle d'un objet socialement construit. Les réflexions qui découlent de cette évolution concernent d'abord la fonction du curriculum comme opérateur de sélection et de transmission culturelles, avec en toile de fond cette interrogation: les contenus d'enseignement sont-ils le reflet d'un univers culturel existant essentiellement en dehors et en amont de l'école ou au contraire l'expression d'une "culture scolaire " relativement spécifique et autonome? Cette dernière conception a alors conduit les sociologues qui la partageaient à s'interroger sur les déterminants et les caractéristiques de cette "culture scolaire", avec en corollaire une réflexion sur la variabilité curriculaire intra- et inter-systèmes éducatifs. Une autre réflexion a porté sur la dimension politique de l'élaboration du curriculum : qui dispose du pouvoir dans les phases d'élaboration puis d'implémentation des curricula? Quel rôle ont les " agents intermédiaires » (inspecteurs, formateurs...) ? De quelles marges de manœuvre disposent en définitive les enseignants? Enfin, le curriculum a aussi été envisagé en termes de gestion, de différenciation, d'ordonnancement social des élèves: quels parcours pour quels élèves?

De façon complémentaire à la sociologie du curriculum-également qualifiée de «nouvelle sociologie de l'éducation »- dont les apports les plus originaux viennent d'être esquissés sur base de la synthèse proposée par Forquin (2008), l'éclairage des «nouvelles approches sociologiques des organisations » (Amblard, Bernoux, Herreros \& Livian, 2005) va également permettre de comprendre les choix curriculaires des enseignants d'EPS. Les actions sociales - et l'élaboration des curricula en est une (cf. paragraphe précédent)-y sont interprétées comme la résultante de deux dimensions: (a) celle de l'acteur, lui-même appréhendé sur toutes les dimensions (social-historique chez Bourdieu, stratégique chez Crozier, identitaire chez Sainsaulieu, etc.) ; (b) celle de la situation d'action, en tant que contexte historique et institutionnel mais aussi d'espace symbolique et mythique. C'est de la confrontation de ces deux dimensions et de leurs composantes, dans ce qu'elles ont de spécifique aux acteurs du système scolaire, qu'émergera l'intelligibilité des choix curriculaires opérés par les enseignants d'EPS. Jusqu'à quel point ces derniers sont-ils contraints ou, à l'inverse, libres dans leurs choix? En corollaire, doit-on les considérer comme des agents, des acteurs ou des sujets (Bajoit, 2010)?

Enfin, nous ajoutons à ce cadrage théorique le modèle de l'activité de l'enseignant proposé par Goigoux (2007). En effet, bien que cet auteur situe sa contribution au croisement de la psychologie ergonomique et de la didactique, il nous semble que son modèle comporte une dimension sociologique non négligeable, notamment par sa prise en compte du contexte dans lequel agit l'enseignant et de la rationalité de ce dernier. Ce modèle repose sur trois entrées, qui représentent les principaux déterminants du travail enseignant (figure 2): (a) les caractéristiques de l'école (cadre institutionnel, prescription, contexte socio-politique, etc.); (b) les caractéristiques des enseignants (finalités et objectifs, conceptions, valeurs et croyances, expérience, etc.); et (c) les caractéristiques des élèves (leurs savoirs et les rapports qu'ils entretiennent avec les savoirs et l'École, leurs compétences, etc.). Il comporte également trois sorties, qui représentent les effets de ce travail et correspondent aux principales orientations de 
celui-ci : (a) vers les autres acteurs du milieu scolaire (parents d'élèves, hiérarchie, collègues, divers partenaires de la co-éducation); (b) vers l'enseignant lui-même (sur le plan physique, psychique, coûts et bénéfices de l'activité, etc.) ; et (c) vers les élèves (individus, classes). Ce modèle peut être considéré comme cyclique dans la mesure où les effets produits par le travail enseignant sont susceptibles de jouer, à plus ou moins long terme, un rôle déterminant pour l'activité future. Par ailleurs, l'étude de la tâche redéfinie est un élément central du modèle, ce qui « implique de s'intéresser à la manière dont ceux-ci [les enseignants] interprètent les prescriptions en fonction des moyens dont ils disposent et des exigences qu'ils se donnent" (p.56). Enfin, ce modèle accorde au genre professionnel (Clot, 1999, cité par Goigoux, 2007) - entendu comme «un ensemble de schèmes sociaux [...], un corps d'évaluations partagées qui règle l'activité personnelle de façon tacite et marque l'appartenance à un groupe " (Goigoux, 2007, p. 58)-un statut d'intercalaire entre le travail prescrit et le travail réel. Il postule en outre que l'activité de l'enseignant est une activité médiatisée par des instruments didactiques.

Figure 2. Modèle d'analyse de l'activité de l'enseignant (Goigoux, 2007)

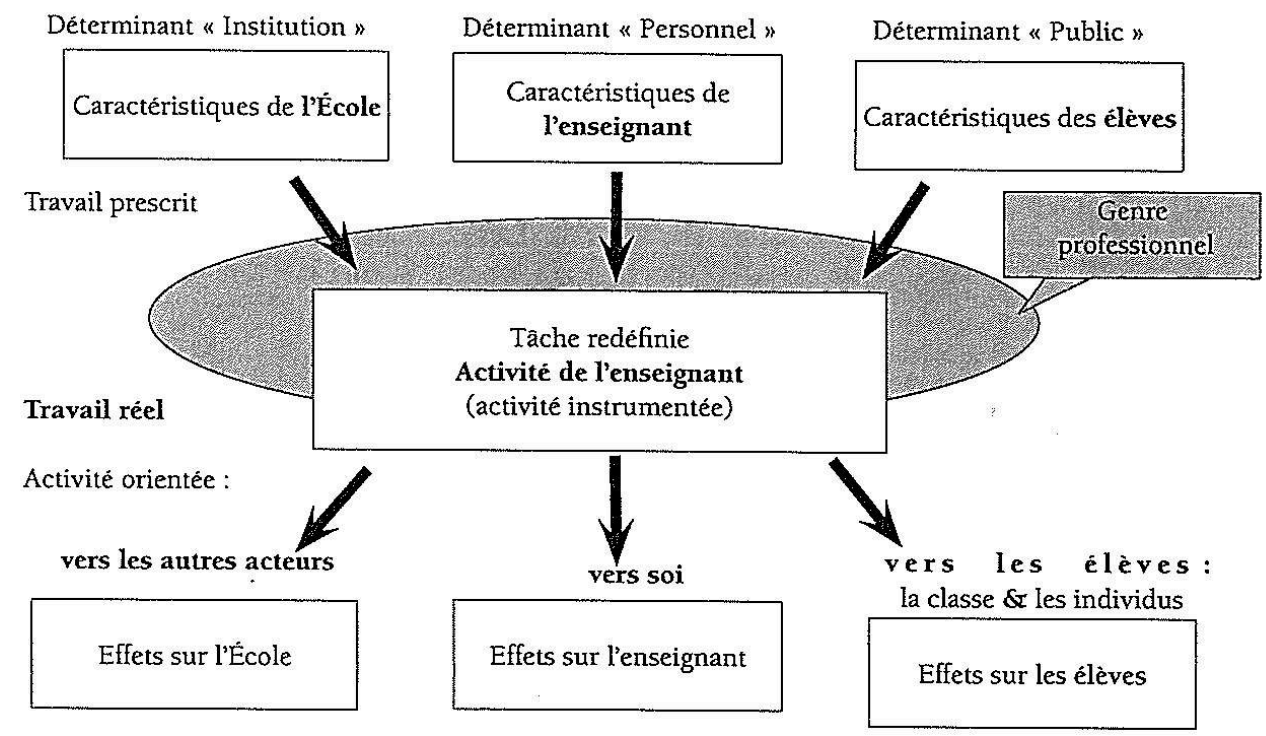

Dans le même ordre d'idées, Briot (1999) avait cherché à dépasser la dichotomie théorique traditionnelle curriculum formel/curriculum réel, jugée insuffisamment adaptée à la complexification des lieux de réflexion et des références curriculaires, dont témoigne le modèle de Perrenoud (1998). Elle avait dès lors proposé une structuration des références curriculaires en trois niveaux: (a) le niveau macrostructurel (plan supérieur officiel de type prescriptif), celui des programmes et instructions officielles; (b) le niveau méso-structurel (plan intermédiaire structurel de type incitatif), celui des projets d'établissement et d'équipe pédagogique, des structures de formation continue ; (c) le niveau micro-structurel (plan terminal individuel de type actualisé), celui des enseignants. De notre point de vue, le déterminant «Institution » du modèle de Goigoux relève à la fois d'influences macro- et méso-structurelles, se voyant dès lors affiné grâce à cet apport. Le déterminant « Personnel » du modèle de Goigoux relève quant à lui du niveau d'influence micro-structurel. Enfin, les caractéristiques des élèves (déterminant «Public » du modèle de Goigoux) sont incluses dans les références méso-structurelles de la modélisation proposée par Briot. Par conséquent, nous retenons le modèle de Goigoux, ainsi complété par celui de Briot, 
pour contribuer à éclairer le comment et le pourquoi des décisions curriculaires des enseignants d'EPS.

\section{Questions de recherche}

En cohérence avec ce cadre théorique socio-didactique, les questions de recherche auxquelles nous allons tenter d'apporter des réponses à travers cette revue de littérature sont les suivantes: (a) quels contenus résultent des choix opérés par les enseignants d'EPS (quelles références? Univers culturel existant en dehors de l'école et dans ce cas quelle distance entre celui-ci et les contenus proposés par les enseignants ? - ou «culture scolaire»?)? (b) Comment ces choix sont-ils effectués (sous quelles influences? Avec quelles marges de liberté ?)?

\section{Méthodologie}

7 Dans un premier temps, nous avons constitué un corpus susceptible de s'inscrire dans ce double cadre théorique, en utilisant les mots clés qui nous paraissaient les plus représentatifs de ce dialogue inter-disciplinaire. Le travail de recension a été effectué le 07.02.2011 à partir des bases de données ERIC, Francis et Medline (via CSA), PsycINFO (via HEBSCOhost) et Cairn.info. Il a été complété par une recherche manuelle sur l'ensemble des numéros de la présente revue, non encore indexée dans les bases de données informatisées. Les bases de données ont été choisies, parmi celles auxquelles nous avions accès, pour leur représentativité dans les champs des sciences humaines et sociales francophones et anglophones. La raison de notre choix de consulter la littérature anglophone est double. D'une part, à l'inverse du cadre théorique didactique qui est exclusivement francophone, le cadre théorique sociologique se retrouve à la fois dans la littérature francophone et anglophone. D'autre part, les chercheurs francophones publient également dans les revues anglophones, car celles-ci sont plus nombreuses à être reconnues dans l'évaluation des publications des chercheurs (Cloes, Lenzen \& Trudel, 2009). Le tableau I présente la procédure de constitution du corpus, incluant les mots-clés utilisés et le nombre d'articles ayant franchi les différentes étapes de sélection. Des 239 articles identifiés dans l'étape $n^{\circ} 1$, seuls 62 ont finalement été jugés éligibles (étape $n^{\circ} 3$ ) pour cette revue de question.

Tableau I. Processus de constitution du corpus

\begin{tabular}{|l|l|l|l|l|l|}
\hline & & $\begin{array}{l}\text { ERIC, } \\
\text { Francis }\end{array}$ & PsycINFO & \\
l'étape & et nom de & (HEBSO- & Cairn.info & eJRIEPS \\
& Critères de sélection & $\begin{array}{l}\text { Medline } \\
\text { (CSA) }\end{array}$ & host) & & \\
& & & & \\
\hline
\end{tabular}




\begin{tabular}{|c|c|c|c|c|c|}
\hline \multirow{3}{*}{$\begin{array}{l}1 . \\
\text { Identification }\end{array}$} & $\begin{array}{l}\text { - Mots-clés : ("éducation physique" } \\
\text { or "physical education") } \\
\text { (sociolog* or didact*) } \\
\text { ("transposition didactique" } \\
\text { curricul* or }^{*} \text { or conten*) } \\
\text { (enseignant* or teacher* } \\
\text { profess*) } \\
\text { - Support de publication : revues }\end{array}$ & 40 & 16 & & \\
\hline & $\begin{array}{l}\text { - Mots-clés : ("éducation physique" } \\
\text { and sociologi* or didactique) and } \\
\text { ("transposition didactique" or } \\
\text { curricul* or "contenu* } \\
\text { d'enseignement") } \\
\text { - Support de publication : revues }\end{array}$ & & & 161 & \\
\hline & - Sélection manuelle & & & & 22 \\
\hline 2. Sélection & $\begin{array}{l}\text { - Langue française ou anglaise } \\
\text { - Expertise par des pairs } \\
\text { - Études expérimentales ou méta- } \\
\text { analyses }\end{array}$ & 29 & 14 & 88 & 22 \\
\hline 3. Éligibilité & $\begin{array}{l}\text { - Pas d'intervention } \\
\text { - Éducation physique « ordinaire» } \\
\text { - Suppression des doublons } \\
\text { - Réponse à l'une des deux } \\
\text { questions de recherche au moins }\end{array}$ & 7 & 1 & 33 & 21 \\
\hline \multirow{5}{*}{$\begin{array}{l}4 . \\
\text { Catégorisation }\end{array}$} & Cadre théorique didactique & 19 & & & \\
\hline & Cadre théorique sociologique & 10 & & & \\
\hline & Cadre théorique socio-didactique & 3 & & & \\
\hline & Autre cadre théorique & 23 & & & \\
\hline & Pas de cadre théorique & 7 & & & \\
\hline
\end{tabular}

8 Ces études se réfèrent à divers cadres théoriques. Un peu plus de la moitié d'entre elles (32/62) s'inscrivent dans un cadre théorique soit didactique (19), soit sociologique (10), soit socio-didactique (3). Vingt-trois études se réfèrent à d'autres cadres théoriques (cours d'action, psychologie, psychosociologie, etc.) que celui qui oriente cette revue de littérature. Enfin, nous avons catégorisé sept études comme étant a-théoriques.

Dans un second temps, nous avons synthétisé dans un tableau, pour chacune de ces 62 études, les données qui apportaient des éléments de réponse à l'une, l'autre ou les deux questions de recherche précédemment définies. Ce sont ces deux questions de recherche qui structureront la section suivante, consacrée aux résultats de notre analyse. 


\section{Résultats}

\section{1. Quels contenus résultent des choix opérés par les enseignants d'EPS?}

9 La majorité des études recensées renvoient à l'idée que les contenus enseignés en EPS sont très largement référés à un univers culturel existant en dehors de l'école, plus précisément aux activités physiques et sportives (APS) institutionnellement organisées par les fédérations et associations sportives. Ce constat nécessite néanmoins d'être affiné et nuancé. Ainsi, chez les 20 enseignants d'EPS tunisiens observés par Lachheb (2008), les pratiques sportives institutionnalisées constituaient l'objet unique de l'enseignement, avec une centration plus prononcée sur l'athlétisme et les sports collectifs. Ces APS prédominaient également, avec la gymnastique et la natation, parmi les disciplines sportives que les 531 enseignants français interrogés par Poggi-Combaz (2002a) déclaraient enseigner. A l'inverse, dans cette même enquête, enseigner une activité d'expression ou de pleine nature constituait une option marginale. De la même façon, les résultats obtenus par Combaz et Hoibian $(2008,2009)$ auprès de 1317 enseignants d'EPS témoignaient de l'existence d'une hiérarchisation des APS, certaines comme la natation, les sports collectifs et l'athlétisme étant jugées incontournables, ainsi que d'une nette préférence pour les formes de pratique institutionnalisées reposant sur l'affrontement et la production de performance. L'enquête réalisée par Marsault (2005) auprès d'une population de 351 enseignants d'EPS aboutissait à des résultats similaires, si ce n'est que le développement fulgurant du badminton dans les établissements scolaires et la diversification des sports collectifs (football américain, base-ball, tchoukball) contribuaient à modifier la définition stricte d'activités traditionnelles liées au milieu scolaire. Vigneron (2006) faisait quant à elle état d'un déclin de la gymnastique par rapport aux études précitées. A l'opposé de cet attachement manifeste des enseignants tunisiens et français aux pratiques sportives fédérales, des chercheurs britanniques (Green, Smith \& Thurston, 2009) ont relevé, dans le cadre d'un " focus group " réunissant 29 enseignants d'EPS, que ces derniers délaissaient les pratiques fédérales au profit d'activités de loisir moins traditionnelles telles que l'aérobic, le trampoline, les activités de pleine nature, l'aquagym, la plongée libre, le canoë, etc.

En restant à ce niveau macroscopique d'analyse, plusieurs chercheurs ont montré que le curriculum en EPS s'avérait inégalitaire au détriment des filles, les APS les plus présentes étant connotées masculines (Cogérino, 2007; Combaz \& Hoibian, 2008; Couchot-Schiex, 2007 ; Vigneron, 2006). L'univers culturel existant en dehors de l'école auquel se réfèrent la majorité des enseignants d'EPS pour concevoir leurs curricula est donc essentiellement celui des garçons. Mais quelle est la part de cet univers qui est transmise par l'institution scolaire? Quel traitement didactique est réservé à ces pratiques sociales de référence?

Plusieurs études, portant sur de plus petits échantillons d'enseignants et des APS plus ciblées, apportent des éléments de réponse à ces questions. Dans certains cas, l'enseignement de la pratique sociale de référence (basket-ball) à l'école ne faisait pas l'objet d'un traitement spécifique au cadre scolaire, au point que les contenus d'enseignement ne se distinguaient pas de ceux observés dans le cadre fédéral (Frédéric, Gribomont \& Cloes, 2007). Dans d'autres cas (gymnastique), le traitement didactique opéré par l'enseignant visait essentiellement une réduction de la complexité 
et une diminution des contraintes liées à l'aménagement matériel (Brière-Guenoun, 2008), ou une orientation particulière de la pratique, tantôt plus artistique et maîtrisée, tantôt plus acrobatique et risquée (Aubert \& Abdi, 2002). Dans l'étude de Verscheure et Amade-Escot (2004), c'est la dimension séquentielle du curriculum qui ressortait, le cycle de volley-ball étant organisé par l'enseignant en trois grandes étapes progressives de la « construction d'attaque ». L'enseignement de la natation a également fait l'objet de plusieurs études, révélant un élargissement de l'univers culturel de référence à une "culture natatoire polyvalente» incluant des pratiques sociales de référence (nage sportive, nage utilitaire et sécuritaire, aquagym, water-polo, nage synchronisée) dont les logiques internes semblent bien n'avoir comme seul point commun que le milieu aquatique dans lequel elles se déroulent (Auvray, 2009 ; Kossivi, 2009a ; Motter, Maraite \& Cloes, 2007).

D'autres études centrées sur les activités physiques artistiques (APA), l'acrogym et la lutte permettent de développer davantage cette question des frontières perméables voire floues entre des pratiques sociales de référence proches, susceptibles de se mélanger en EPS pour constituer ce qui apparaît alors comme une "culture scolaire " spécifique mais pas autonome pour autant puisqu'assujettie à plusieurs pratiques culturelles existant en dehors de l'école. Ainsi, dans le domaine des APA, les références apparaissent multiples et composites, allant de la danse classique à la danse contemporaine en passant par le hip hop, l'expression corporelle et le mime (BuznicBourgeacq, Terrisse \& Margnes, 2007 ; Mons, 1992 ; Thorel, 2009 ; Thorel \& David, 2005). Une tension était perçue chez certains enseignants entre la logique de fermeture associée aux activités pseudo-artistiques enfermant les élèves et leur fixant des limites, telles que la danse jazz par exemple, et la logique d'ouverture propre aux pratiques valorisant l'expression libre et le dépassement des limites, telles que la danse contemporaine par exemple (Ferez, 2004). Les références apparaissent tout aussi composites dans l'enseignement scolaire de l'acrosport, pour lequel la majorité des enseignants peut difficilement se référer à la pratique fédérale dans la mesure où ils n'en ont qu'une idée assez vague (Musard, Robin \& Caty, 2009 ; Musard, Robin, Nachon \& Gréhaigne, 2008). Dès lors, les enseignants ne cherchent pas à transposer la pratique sociale de référence mais associent l'acrosport à d'autres activités (gymnastique, cirque, danse). Le recours à d'autres références que celle propre à l'activité enseignée a également été mis en évidence chez une enseignante d'EPS stagiaire qui incorporait des savoirs et des pratiques issus du judo dans son enseignement de lutte (BuznicBourgeacq et al., 2007). Ces derniers auteurs parlent de « conversion didactique " pour désigner cette conversion de l'expérience du sujet en objets de savoirs enseignés, qui a pour résultat des formes de pratiques scolaires qui n'ont pas d'équivalent strict en dehors de l'école.

Qu'elles se réfèrent de manière univoque à un univers culturel bien identifié en dehors de l'école ou simultanément à plusieurs pratiques sociales distinctes, les pratiques scolaires en EPS se caractérisent quasiment toutes par une prise de distance vis-à-vis des pratiques extrascolaires, pouvant aller de simples aménagements en fonction des impératifs éducatifs jusqu'à ce qui apparaît comme des ruptures avec la logique interne de la pratique sociale de référence. Le traitement didactique réservé aux pratiques sociales de référence pour les "apprêter » en vue de leur enseignement à l'école se caractérise également par la prise en compte de ce qu'il est convenu d'appeler les " savoirs d'accompagnement » : échauffement, arbitrage, aide, conseiller, observateur, etc. (Cogérino, 2002). Ainsi, Baeza, Nourrit-Lucas et Bouthier (2009) ont catégorisé 
comme suit ce qu'il y avait à apprendre dans deux leçons de lutte : mettre en place des surfaces de travail sécurisées, apprendre des techniques de contrôle, énoncer des règles de sécurité, incorporer les rôles sociaux, comprendre la notion de déséquilibre des corps, appréhender le poids de son adversaire, contrôler le corps de l'autre, donner une direction à son corps et arbitrer un combat. Il ne s'agit donc pas seulement pour les élèves d'apprendre à lutter, mais également d'acquérir des compétences propres à d'autres acteurs du milieu de la lutte que les lutteurs eux-mêmes (entraîneurs, arbitres, responsables du matériel). Comparant l'enseignement dispensé par les membres de l'équipe d'EPS dans deux collèges comparables, Briot et Chifflet (2001) ont relevé dans l'un des deux collèges une prise en compte par l'ensemble des enseignants de ces "savoirs d'accompagnement»: auto-arbitrage, auto-échauffement, auto-évaluation, participation de chaque élève à la prise en charge du matériel, alors que ce n'était pas le cas dans l'autre. Pour leur part, David et Amarouche (2006) ont mobilisé la technique d'autoconfrontation dite " des instructions au sosie » afin d'approfondir dans le détail l'activité évaluative en classe de trois enseignants stagiaires d'EPS. Une enseignante en ZEP y déclarait initier notamment les élèves de $6^{\text {ème }}$ à l'utilisation du chronomètre et au départ, dans le souci principal d' "occuper les élèves perturbateurs". Analysant les pratiques effectives de quatre enseignants d'EPS spécialistes de judo, Loizon, Margnes et Terrisse (2008) ont noté que ces derniers transmettaient à leurs élèves, outre des savoirs techniques, stratégiques, règlementaires et culturels, d'autres objets de savoirs en lien avec les missions éducatives de l'institution scolaire, comme les savoirs sécuritaires et éthiques. Enfin, même s'ils formaient leurs élèves à l'observation de leurs condisciples, la majorité des professeurs stagiaires observés par Carnus et Terrisse (2006) assuraient l'essentiel de l'évaluation sans trop déléguer aux élèves. Lorsqu'ils le faisaient malgré tout, ils prenaient soin de doubler l'évaluation réalisée par les élèves par une évaluation propre.

\section{2. Comment les enseignants d'EPS effectuent-ils ces choix ?}

Pour structurer la deuxième partie des résultats, nous nous appuierons sur le système de catégories du modèle de Goigoux (2007) complété par celui de Briot (1999), en nous limitant aux déterminants et au genre professionnel dans la mesure où les effets de l'activité de l'enseignant sortent du cadre de cette revue de littérature et ne sont pas susceptibles de trouver un écho dans le corpus que nous avons constitué à cet effet.

\section{2. 1. Le déterminant «Institution»}

11 Les instructions officielles (textes légaux, programmes, plans d'études...) constituent le niveau le plus institutionnel et prescriptif parmi les trois niveaux de références curriculaires (macro-, méso- et micro-structurels) distingués par Briot (1999). Pourtant, la majorité des études traitant de ce déterminant institutionnel aboutissent au constat d'un faible impact des instructions officielles sur les décisions curriculaires des enseignants d'EPS. Ainsi, en France, moins de la moitié des 102 enseignants d'EPS interrogés par Musard et ses collaborateurs (Musard et al., 2008, 2009) déclaraient se référer aux programmes d'EPS pour construire leurs curricula en acrosport. Lors d'entretiens menés par Cordoba (1996) auprès d'enseignants généralistes et d'EPS qui se partageaient l'enseignement de l'EPS à l'école primaire dans le canton de Genève en Suisse, ces derniers avouaient ne pas se référer au curriculum formel et construire la 
discipline en toute liberté, cette liberté étant facilitée par l'absence de contrôle institutionnel de leur enseignement en classe. En revanche, cette liberté était malgré tout contrainte par le fait que les enseignants devaient présenter leur planification annuelle au chef de service qui acceptait ou non ce qui était prévu, et par le fait qu'ils étaient animés par l'envie "d'être couverts " au niveau légal. Au Québec, alors que le programme d'études provincial de 1981 valorisait le développement des actions de coopération et d'opposition simultanées, thème associé à l'utilisation des sports collectifs et du badminton en double, les sept cycles d'enseignement dans ces APS observés au secondaire par Genet-Volet et Desrosiers (1995) offraient très peu de contenus tactiques ou stratégiques susceptibles de rencontrer les prescriptions du programme. Au Québec toujours mais en référence cette fois à des instructions ministérielles plus récentes (2001 pour l'enseignement préscolaire et primaire) se traduisant par une modification du rôle de l'éducation physique en incluant dans ses objets d'enseignement la compétence " adopter un mode de vie sain et actif », Turcotte, Otis et Gaudreau (2007) ont constaté que, lorsqu'elle était incluse en éducation physique au primaire, l'éducation à la santé avait pour principal objet d'enseignementapprentissage l'amélioration de la condition physique. Les dix enseignants observés ne mettaient que très partiellement en œuvre le nouveau programme, lequel prescrivait l'enseignement de plusieurs savoirs jugés indispensables à l'éducation à la santé (pratique régulière de l'activité physique, relaxation, hygiène corporelle, effets de la sédentarité, gestion du stress, etc.), que la majorité de ces enseignants négligeaient au profit d'autres ne figurant pas dans le programme (alimentation, premiers soins, éthique sportive, etc.). Cogérino, Marzin et Méchin (1998) avaient également relevé en France que la course de longue durée tenait majoritairement lieu de prévention sur le terrain de la santé physiologique, concluant que l'introduction de nouvelles injonctions officielles (en l'occurrence ici l'apparition en 1993 de l'objectif "gérer sa vie physique ») n'entraînait pas directement de modifications dans les curricula. Le même constat a été fait quelques années plus tard par Cogérino (2002) suite à la promulgation de nouvelles instructions officielles, relatives cette fois à l'évaluation des «savoirs d'accompagnement » entrée en vigueur en 1995.

Cette inertie manifeste des curricula lors de l'introduction de nouvelles directives pourrait en partie s'expliquer par une tendance des enseignants d'EPS à interpréter les textes à leur avantage, c'est-à-dire à trouver dans les textes de quoi légitimer leurs pratiques habituelles, voire pour certains à ne les lire que partiellement ou en surface pour satisfaire aux exigences minimales (Roux-Perez, 2004). Certaines orientations de valeur des enseignants (déterminant «Personnel», voir 4.2.2.) pourraient aussi les pousser à résister aux changements impulsés par le curriculum officiel (Pasco \& Ennis, 2009). Des impossibilités structurelles peuvent également expliquer la non prise en compte des instructions officielles par les enseignants d'EPS. Ainsi, dans des contextes d'enseignement moins privilégiés tels qu'au Bénin par exemple, les instructions officielles prescrivaient l'enseignement de certaines APS (natation, volley-ball, basketball) alors que la plupart des écoles secondaires n'étaient pas dotées d'infrastructures permettant leur pratique et donc leur enseignement. En outre, l'effectif souvent pléthorique des classes (50 à 80 élèves !) ne permettait pas l'application de la démarche d'enseignement/apprentissage préconisée par les programmes (Kossivi, 2009b).

Ce constat d'un faible impact des instructions officielles sur les décisions curriculaires des enseignants d'EPS doit toutefois être nuancé. Dans certains contextes, dont celui dans lequel s'est déroulé l'enseignement de la natation en France entre 1945 et 1995, 
l'imprécision ou la largesse des curricula formels offrent le plus souvent la possibilité aux enseignants d'implémenter des curricula réels très hétérogènes mais toujours en conformité avec le mandat prescrit (Auvray, 2009). Cela ne semble plus être le cas avec les nouveaux programmes de lycée en France, qui détaillent désormais pour chaque APSA les contenus d'enseignement, mais c'est encore le cas dans bien d'autres systèmes scolaires, à l'exemple de celui du Bénin (Kossivi, Minakpon Albert, Souaïbou, Anicet \& Reffugi, 2006) et d'autres régions que nous connaissons (Belgique francophone, Suisse romande), où les programmes et autres plans d'études demeurent très généraux. Par ailleurs, les injonctions officielles, particulièrement celles des programmes d'EPS, peuvent être intériorisées par certains enseignants (Brière-Guenoun, 2008). D'autre part, les programmes étant eux-mêmes, en France en tout cas, influencés par une dimension institutionnelle issue des fédérations sportives nationales et internationales (Combaz \& Hoibian, 2009; Hsouna, Terrisse \& Berhaim, 2007), adhérer et se conformer à la culture sportive fédérale peut impliquer de facto une certaine conformité aux instructions officielles alors que celle-ci n'est pas recherchée systématiquement par les enseignants. Cela se vérifiait notamment dans l'étude de cas réalisée par Heuser (2008) auprès d'un enseignant d'EPS spécialiste du karaté. Le savoir à enseigner de cet enseignant se positionnait clairement dans une optique duo/duel, faisant partie de la culture du karaté tout en étant prônée par les programmes dans les activités de combat au lycée. Toutefois, une étude tranche nettement par rapport aux données plutôt nuancées synthétisées ci-avant. Ayant observé 20 leçons d'EPS se déroulant dans huit collèges de la région du Grand-Tunis, Lachheb (2008) affirmait que tous les enseignants y appliquaient les directives consignées dans les instructions officielles et les programmes. La formation initiale des enseignants ainsi qu'une politique très centralisée - l'enseignement de l'EPS est obligatoire et identique sur tout le territoire tunisien - semblaient jouer un rôle déterminant dans l'homogénéité marquante caractérisant les leçons observées, ce qui faisait dire à Lachheb que le nombre de séances observées apparaissait suffisant pour refléter la réalité, « car les caractéristiques des pratiques enseignantes observées se répètent » (p. 151).

Parmi les structures plus locales ayant valeur incitative sur le plan des contenus d'enseignement (niveau méso-structurel), celle de l'équipe pédagogique exerce une influence très particulière, qualifiée d'« effet équipe d'EPS » (Briot, 1999). En comparant les curricula en EPS dans deux collèges aux caractéristiques très comparables, Briot et Chifflet (2001) ont relevé à l'intérieur de chaque établissement un ensemble de ressemblances au niveau des contenus d'enseignement qui relevait de l'action organisée au sein de l'équipe d'EPS et qui différait d'une équipe à l'autre en fonction de l'intensité de l'action organisée. Cette intensité dépendait elle-même de facteurs contextuels, notamment « l'histoire » de l'équipe. La présence d'un leader, la pérennité de l'équipe et le soutien apporté par l'établissement dans son ensemble au travail effectué par l'équipe d'EPS potentialisent quant à eux l'« effet équipe d'EPS » (Briot \& Chifflet, 2001; Garnier, 2008). Lorsque la mémoire et les habitudes de l'équipe se retrouvent consignées dans de véritables projets d'établissement, ces projets exercent alors une influence non négligeable sur les curricula en EPS au sein de ces établissements (Baeza \& Nurit-Lucas, 2009; Kossivi, 2009a). Un quart des enseignants interrogés par Musard et al. (2008, 2009) déclarait ainsi s'appuyer sur les projets d'établissement et d'EPS pour construire leurs curricula en acrosport. Ce déterminisme des projets d'établissement se vérifie également a contrario: l'absence de projets d'établissement en judo constituait un obstacle majeur à l'enseignement de cette APS 
au Bénin (Kossivi et al., 2006).

Enfin, quelques autres déterminants de type «Institution » ont été relevés dans notre corpus, à commencer par les structures de formation continue (niveau mésostructurel). Plus de la moitié des enseignants interrogés par Musard et al. $(2008,2009)$ affirmait s'appuyer sur la formation continue pour élaborer leurs curricula en acrosport. Dugas (2009) avait quant à lui constaté une évolution des représentations des enseignants suite à un stage de formation continue centré sur la programmation des jeux traditionnels en EPS. A noter que le cheminement des savoirs, du savoir expert aux savoirs incorporés à « l'épistémologie des professeurs » en passant par les savoirs mis en jeu dans l'action de formation continue, peut se révéler parsemé d'obstacles. Ainsi, il ne faut pas sous-estimer le champ de détermination qui pèse sur des enseignants dont l'épistémologie professionnelle est travaillée par deux institutions (scolaire et sportive) du fait de leurs activités parallèles d'enseignant d'EPS et d'entraîneur. La difficulté de passer de l'une à l'autre avec des contraintes de nature différente peut générer un manque de perméabilité à l'épistémologie renouvelée voulue par la formation continue (Vandevelde \& Amade-Escot, 2003). Les contraintes matérielles ont déjà été mentionnées en tant que facteur limitant les choix curriculaires des enseignants d'EPS dans des contextes peu dotés en infrastructures, ce qui est toujours le cas au Bénin aujourd'hui (Kossivi, 2009a, 2009b) et ce qui était le cas en France durant la période 1945-1970 s'agissant de l'enseignement de la natation (Auvray, 2009). Néanmoins, les résultats de Poggi-Combaz (2002a) permettent de relativiser la portée de ce déterminant dans les conditions d'enseignement actuelles des pays industrialisés. En effet, en interrogeant les enseignants d'EPS sur un « curriculum idéal » ou " souhaité », imaginé à partir d'une situation où les conditions matérielles ne constitueraient pas une contrainte, elle a montré que globalement, ce curriculum s'alignait sur le curriculum que ces enseignants déclaraient réellement mettre en œuvre. Parmi les déterminants institutionnels des curricula en EPS, on peut encore citer la littérature professionnelle, que les enseignants déclaraient essentiellement consulter pour varier leur enseignement (Cordoba, 1996) ou pallier les lacunes de leur formation initiale en matière d'APSA (Musard et al., 2008, 2009) ou de nouvelles finalités de l'EPS (Turcotte et al., 2007). Pour terminer, si les chefs d'établissement déclaraient exercer une influence sur l'enseignement de l'EPS dans les domaines extrascolaire, organisationnel et disciplinaire, ils déclaraient en revanche ne pas intervenir dans le champ pédagogique spécifique à l'EPS en fonction des compétences qu'ils reconnaissaient à leur équipe. L'influence moyenne déclarée par les chefs d'établissement possédant une formation en EPS était toutefois nettement plus élevée que celle des autres chefs d'établissement (Mees, Carlier \& Renard, 2003).

\section{2. 2. Le déterminant «Personnel»}

12 Les études de notre corpus font ressortir plusieurs caractéristiques déterminantes des enseignants d'EPS (niveau micro-structurel), à commencer par leurs représentations des APSA enseignées, dont plusieurs auteurs ont montré l'influence sur les curricula (Aubert \& Abdi, 2002; Garnier, 2008; Kossivi, 2009a ; Vigneron, 2006). Garnier et Amade-Escot (2003) faisaient notamment état d'une tension chez un enseignant d'EPS entre l'injonction scolaire de privilégier une gymnastique peu aérienne (dans le programme, le terme de posture, de position revenait fréquemment) et la référence privée à une gymnastique aérienne en élan. De son côté, Brau-Antony (2001) mettait en 
évidence l'aspect composite, voire parfois contradictoire, des conceptions des enseignants d'EPS en matière d'enseignement des jeux sportifs collectifs. Celles-ci n'obéissaient pas aux mêmes logiques de construction que celles des experts, bien plus homogènes, ce qui laissait présager des curricula assez hétérogènes. Au-delà des aspects propres à la logique interne des APSA enseignées, la traduction personnelle des valeurs éducatives des APSA s'avère déterminante pour décider de leur sélection ou non dans un curriculum réel, puis de leur mise en forme (Poggi, 2007; Poggi-Combaz, 2002a). Avant de subir un traitement didactique ou pédagogique, les APSA subissent de la part des enseignants d'EPS un traitement éducatif, contribuant à une orientation particulière de la pratique vers un objet de savoir particulier (Marsault, 2005). Une étude exploratoire menée auprès de 114 enseignants d'EPS chiliens montrait que les conceptions majoritairement partagées par ces derniers renvoyaient à un modèle d'EPS centré sur la pratique sportive, alors que les programmes prescrivaient un autre modèle, axé sur le développement de l'action motrice (Araya-Cortez \& Carlier, 2005). À noter que les conceptions qu'ont les enseignants d'EPS des APSA qu'ils enseignent et/ ou des finalités de leur discipline scolaire ne sont pas immuables. Roux-Perez (2005) a ainsi témoigné de cette évolution temporelle, résultant à la fois (a) des événements, des changements, des moments de crises et des recompositions, (b) des modes de relation à autrui (élèves, enseignants d'EPS dans l'équipe, autres enseignants, chefs d'établissement), et (c) du rapport à l'institution et à ses recommandations (inspecteurs, textes officiels). Toutefois, même si certains éléments personnels ou contextuels se modifient, l'individu semble conserver une " permanence à soi ». Attard et Armour (2005) ont quant à eux souligné la nécessité de réfléchir sur sa pratique enseignante et de la comparer à celle des autres pour évoluer.

Jewett et Bain (1985, cités par Pasco \& Ennis, 2009) ont introduit la notion d'orientation de valeur pour désigner les préférences des enseignants, lesquelles constituent une base d'orientation agissant comme un filtre qui oriente leurs décisions et leurs actions. Ces auteurs ont caractérisé cinq orientations de valeur propres à l'EPS : la maîtrise de la discipline, le processus d'apprentissage, l'auto-actualisation, la reconstruction sociale et l'intégration écologique. Pour un approfondissement de ces orientations de valeur et pour les résultats de recherches montrant leur influence sur les contenus enseignés en EPS et l'interprétation du curriculum officiel dans cette discipline scolaire, nous renvoyons à la revue de littérature réalisée récemment par Pasco \& Ennis (2009). En résumé, ces résultats montrent de façon convergente que les enseignants d'EPS centrés sur des orientations de valeur différentes enseignent des contenus différents, en cohérence avec leurs priorités. Le profil d'orientations de valeur des enseignants agit comme une "grille de lecture" des perspectives contenues dans les instructions officielles (déterminant "Institution», voir 4.2.1.), toutes ne cadrant pas avec les priorités des enseignants.

D'après Delignières et Garsault (1999), les enseignants d'EPS peuvent être considérés comme des «maîtres polyvalents » amenés à enseigner une dizaine de matières, dont certaines qu'ils maîtrisent moins bien que d'autres. La façon dont l'enseignant conçoit et traite l'APSA qu'il enseigne est apparemment affectée par ses connaissances didactiques spécifiques à cette APSA (Amade-Escot, 2000). Les connaissances disciplinaires des enseignants d'EPS, déclinées sous deux modalités contrastées (spécialisation/non spécialisation ou polyvalence), ont été mises en relation avec les curricula qu'ils proposaient, dans plusieurs études de cas portant sur un à quatre sujets. L'influence de la spécialisation se traduisait notamment par des modalités 
d'organisation du travail et une capacité à faire évoluer l'enseignement en fonction de l'activité des élèves différentes (Adé, Sève \& Serres, 2007). Elle se manifestait aussi dans la quantité de savoirs transmis aux élèves, celle-ci se révélant plus importante dans l'APSA de prédilection (Buznic-Bourgeacq, Terrisse \& Lestel, 2008 ; Buznic-Bourgeacq et al., 2007). Enfin, le contenu des enseignements dans la spécialité s'avérait beaucoup plus ouvert, dynamique et complexe, avec des situations moins didactisées et plus proches des conditions effectives de la pratique sociale de référence (BuznicBourgeacq, Terrisse \& Margnes, 2010). Par ailleurs, Hsouna et al. (2007) ont noté une tendance des enseignants à se référer davantage au programme officiel (déterminant "Institution », voir 4.2.1.) pour l'enseignement de la non spécialité. Cette stratégie peut être rapprochée de celle observée par Rovegno (2004) chez deux enseignants stagiaires, consistant, lorsqu'ils se sentaient en difficulté, à se retrancher dans ce que Rovegno a appelé une " zone curriculaire de sécurité » (" curricular zone of safety »), c'est-à-dire à se limiter à l'utilisation de situations d'apprentissage qui ne rompent pas avec leurs habitudes et ne sont exigeantes ni pour eux-mêmes, ni pour leurs élèves.

Parmi les caractéristiques des enseignants d'EPS, la formation qu'ils ont reçue est évidemment susceptible d'exercer une influence sur leurs pratiques curriculaires. La même formation ne bénéficie toutefois pas de la même façon à tous ceux qui la reçoivent, ainsi qu'en témoignent les apports variables d'une formation à l'évaluation inférés des pratiques évaluatives de quatre enseignants stagiaires (Carnus \& Terrisse, 2006). Brière-Guenoun (2008) avait quant à elle retrouvé la trace de l'assujettissement d'un enseignant d'EPS à sa formation initiale (et notamment à la préparation au concours), qui s'exprimait non seulement à travers le discours tenu, mais également à travers les contenus et les registres de savoirs activés dans son enseignement de gymnastique. Ce déterminisme relatif de la formation se vérifie également $a$ contrario : le fait que même s'ils avaient été formés au judo, les enseignants d'EPS béninois n'avaient jamais été amenés à l'enseigner en formation, constituait un obstacle majeur à l'enseignement de cette APS dans les écoles béninoises (Kossivi et al., 2006). Par ailleurs, si la formation amène des connaissances aux enseignants d'EPS, elle génère aussi son lot d'incertitudes, ainsi qu'en atteste le nombre élevé de réponses " ne sait pas » à une question portant sur les principes généraux qui organisent la préparation physique intégrée à l'échauffement dans l'étude de Maquaire (2007) sur la place des étirements dans l'échauffement en EPS. Cette étude illustre également le décalage des pratiques enseignantes avec l'avancée des connaissances scientifiques dans la littérature spécialisée.

Le genre des enseignants d'EPS (construction psychosociale du sexe, à distinguer du sexe comme attribut biologique) a également été considéré comme un facteur susceptible d'interférer dans les décisions curriculaires de ces derniers. Il s'est effectivement avéré déterminant dans l'étude de Couchot-Schiex (2007), portant sur les contenus enseignés par 16 enseignants d'EPS dans trois APSA choisies pour leur connotation sexuée : la gymnastique (féminine), le badminton (appropriée aux deux sexes) et le handball (masculine). Pour les activités badminton et handball, les enseignants catégorisés masculins (deux hommes et deux femmes) proposaient des apprentissages centrés sur les aspects stratégiques alors que les enseignants catégorisés androgynes, non différenciés ou féminins (deux hommes et deux femmes par catégorie) proposaient des apprentissages centrés sur les aspects techniques. Dans le cas de l'enseignement de la danse, le processus de construction curriculaire s'effectuait à partir d'un système d'interaction entre la "position de genre » des 
enseignants et leur univers de danse préférentiel (Thorel, 2009 ; Thorel \& David, 2010). En revanche, aucune relation significative entre les variables de sexe et d'âge des enseignants et les curricula en EPS n'a été relevée dans les études ayant tenté de mesurer séparément l'influence de ces attributs biologiques (Lachheb, 2008 ; Musard et al., 2009). De même, Pasco, Kermarrec et Guinard (2008) ont conclu à l'absence de relation entre le sexe et l'âge des enseignants d'EPS d'une part, et leurs orientations de valeur d'autre part.

Le déterminant «Personnel » de l'activité curriculaire des enseignants d'EPS recouvre donc les représentations, conceptions, orientations de valeur de ces derniers relatives à plusieurs facettes de leur activité professionnelle (APSA de référence, finalités de l'EPS, etc.). Il recouvre également les connaissances des enseignants (e.g., familiarité avec l'APSA enseignée) et la formation dont ils ont bénéficié, ainsi que leur genre, mais apparemment pas leur sexe ni leur âge.

\section{2. 3. Le déterminant « Public»}

13 L'influence des élèves peut s'exercer de manière directe ou indirecte sur les curricula qui leur sont proposés par les enseignants d'EPS. Le premier cas est notamment illustré par l'étude de Musard et al. $(2009,2009)$ dans laquelle $76,5 \%$ des enseignants interrogés déclaraient s'inspirer des idées originales des élèves (création de pyramides ou d'éléments de liaison, utilisation d'accessoires et de musique, expression d'un thème) pour concevoir leurs contenus d'enseignement en acrosport. L'influence directe des élèves ressortait également nettement dans le discours des généralistes enseignant l'EPS à l'école primaire (Cordoba, 1996). Ces dernières - car il s'agissait majoritairement d'enseignantes - avouaient en effet généralement demander aux enfants ce qu'ils souhaitaient faire durant l'heure d'EPS: «on ne peut pas proposer un jeu s'ils n'ont pas envie de le faire... » (p. 7).

Néanmoins, dans la plupart des cas, c'est de manière indirecte que les élèves contribuent à orienter les curricula qui leur sont destinés. Ainsi, dans les séances d'EPS observées par Buznic-Bourgeacq et al. (2007, 2008), bon nombre de savoirs émergeaient in situ face aux obstacles rencontrés par les élèves, sans que leur transmission ait été prévue a priori. La mixité des classes contraint également les choix curriculaires des enseignants d'EPS (Cogérino, 2007 ; Couchot-Schiex, Cogérino \& Coltice, 2009). Pour susciter l'intérêt, certains enseignants étaient ainsi conduits à des " arrangements " à l'intérieur de la logique pédagogique : accepter la construction d'un espace de travail à l'intérieur de l'espace du cours de danse, ou encore des configurations qui permettaient de mettre en valeur les garçons au centre d'un corps de ballet constitué par les filles (Thorel \& David, 2005). Mais c'est surtout la composition sociale du public cible qui a fait l'objet de recherches visant à dévoiler l'influence indirecte, voire involontaire de ce déterminant «Public ». Partant de l'hypothèse que les disparités importantes dans la façon de concevoir et mettre en œuvre les contenus d'enseignement en EPS s'expliqueraient, en partie, par les variations liées aux caractéristiques sociales des publics scolaires, Poggi (Poggi, 2007; Poggi-Combaz, 2002a, 2002b) a interrogé 531 enseignants d'EPS, puis contrasté leurs réponses selon le type d'établissement dans lequel ils enseignaient (défavorisés, très défavorisés, très favorisés). Les résultats valident majoritairement l'hypothèse de départ. Ainsi, la conception de l'orientation dominante de la séance, les modalités de traitement didactique des APS ainsi que les dispositifs mis en place dans le domaine de l'évaluation variaient d'un type 
d'établissement à l'autre. Exercer en établissement très défavorisé diminuait la marge de manœuvre des enseignants et les contraignait à organiser leur enseignement à partir d'un modèle largement dominé par la logique sportive (Poggi, 2007). Les décisions curriculaires des enseignants d'EPS sont donc dépendantes des représentations que ceux-ci se font des caractéristiques de leurs élèves et du pouvoir éducatif des APS (Poggi-Combaz, 2002a). En effet, les contenus d'enseignement n'étaient pas définis uniquement et prioritairement par rapport aux apprentissages moteurs, mais le développement psychologique et social de l'enfant était d'abord mis en avant. La façon de concevoir l'élève oscillait ainsi entre une approche centrée sur "l'enfant citoyen " en établissements très défavorisés et une démarche organisée autour de "l'enfant apprenant» en établissements très favorisés (Poggi-Combaz, 2002b). Les activités pratiquées en milieu incertain étant jugées moins formatrices que celles se pratiquant en milieu standardisé, les élèves des établissements défavorisés avaient par conséquent moins de chance que les élèves d'établissements plus favorisés d'en bénéficier (Poggi-Combaz, 2002a). Debars et Amade-Escot (2006) ont quant à elles étudié l'incidence du contexte ZEP (zone d'éducation prioritaire) sur les pratiques d'intervention d'un enseignant d'EPS lors de trois leçons de badminton. Le souci de contrôler les élèves entraînait un éclatement du contenu initialement pensé pour introduire l'alternative servir court ou servir long, par la mise en place en cours des leçons d'une succession de micro-tâches centrées sur l'effectuation d'un geste.

\section{2. 4. Le genre professionnel}

14 À quoi pourraient correspondre, dans les études de notre corpus, "les 'obligations' que partagent ceux qui travaillent pour arriver à travailler, souvent malgré tout, parfois malgré l'organisation prescrite du travail (...), ces formes communes de la vie professionnelle (...), ce que les travailleurs d'un milieu donné connaissent et voient, attendent et reconnaissent, apprécient ou redoutent (...), comme 'un mot de passe' connu seulement de ceux qui appartiennent au même horizon social et professionnel » qui définissent le genre professionnel selon Clot \& Faïta (2000, pp. 9-11) ? Seules, trois études de notre corpus nous semblent fournir des données de cet ordre. La première est celle de Briot et Chifflet (2001), à l'issue de laquelle ces auteurs concluaient que les conditions inhérentes à cette discipline scolaire particulière qu'est l'EPS (contraintes d'utilisation de lieux et matériels communs, visées stratégiques de confort pédagogique ou de reconnaissance institutionnelle...) expliquaient notamment la présence de contenus d'enseignement similaires entre les équipes d'EPS de deux établissements distincts, indépendamment du type d'action organisée caractérisant le fonctionnement de chacune d'elles. Voici quelques exemples trouvés dans cette étude, qui illustrent selon nous ce qui peut alimenter le genre professionnel des enseignants d'EPS: maintien du même matériel en place sur une demi-journée; travail des enseignants en binôme, facilitant les problèmes de mise en place du matériel et satisfaisant le "besoin d'être rassurés "; reproduction mimétique de ce que fait le collègue dans le même espace de pratique professionnelle. Les deux autres études concernent la gestion de la sécurité, contrainte qui distingue l'EPS des autres disciplines scolaires et est également susceptible, de notre point de vue, de contribuer à la construction du genre professionnel des enseignants d'EPS. BrièreGuenoun (2008) a notamment montré que la conception de certaines tâches d'apprentissage en gymnastique était le résultat de compromis entre des préoccupations sécuritaires et des préoccupations relatives aux apprentissages dans le 
chef de l'enseignant d'EPS. Ainsi, lorsque ce dernier proposait un dispositif «monter rouler » avec trampoline pour préparer les élèves au franchissement par redressement au saut de cheval, il tentait de dépasser des contradictions entre l'aménagement de conditions sécuritaires et les possibilités de renvoi de la surface d'impulsion. Dans une étude historique sur la sécurité des élèves depuis le XXe siècle dans un lycée bisontin, Vivier et Dupaux (2007) ont mis en évidence que l'enseignement de la gymnastique était accompagné d'une double dangerosité : corporelle (menace de l'intégrité physique des élèves) et morale (suspicion d'attouchement de l'enseignant lors d'actions de parade ou d'aide). Ce serait cette double dangerosité qui expliquerait le déclin de la gymnastique dans les écoles, au profit d'activités telles que l'acrogym et le cirque où les risques peuvent sembler moindre et où les interventions physiques de l'enseignant dans les parades peuvent être plus facilement déléguées aux élèves.

\section{Discussion}

Cette section sera structurée de façon similaire à la section précédente, en fonction des deux questions de recherche qui ont orienté cette revue de littérature.

\section{1. Quels contenus résultent des choix opérés par les enseignants d'EPS?}

16 À l'exception de quelques études qui témoignent d'une importation «brute » des pratiques sportives fédérales à l'intérieur des murs de l'école (Frédéric et al., 2009; Lachheb, 2008), les études de notre corpus valident majoritairement l'idée d'une « culture scolaire des activités physiques en EPS » (Delhemme, 2007, cité par Combaz \& Hoibian, 2009), ou dit autrement, d'une mise en forme scolaire des pratiques sociales de référence (Poggi, 2007). Ce constat nécessite maintenant d'être discuté, à la fois en termes de ce qu'il traduit comme pratiques curriculaires et en termes d'éventuelles limites méthodologiques qui nous y ont amenés.

Les pratiques sociales qui servent de référence à l'enseignement de l'EPS sont majoritairement constituées des APS institutionnellement organisées par les fédérations et associations sportives, une certaine hiérarchisation de celles-ci étant parfois opérée par les enseignants. Nous avons néanmoins retrouvé dans plusieurs études d'autres pratiques de référence, telles que les activités physiques de loisir (aérobic, aquagym, plongée libre, etc.) et les pratiques artistiques et d'expression (différents univers de danse dont la danse classique, la danse contemporaine et le hiphop, expression corporelle, mime, etc.). Les curricula en EPS ne sont ainsi jamais totalement déconnectés des pratiques sociales existant en dehors de l'école. Ces pratiques sociales sont parfois mélangées (gymnastique et cirque, lutte et judo...) pour constituer un amalgame qui, en tant que tel, n'a pas d'équivalent extrascolaire. Il ne s'agit pas pour autant de créations didactiques comme on a pu le dire, au moment où ces pratiques typiquement scolaires sont apparues, de la grammaire, "créée par l'école elle-même, dans l'école et pour l'école » (Chervel, 1988, p. 66), ainsi que du volley-ball et du basket-ball, "construits de toute pièce par l'école à la fin du XIXe siècle pour répondre aux valeurs de solidarité et d'entraide» (Poggi et al., 2010, p. 162). C'est précisément pour satisfaire aux missions que l'institution scolaire se donne et assigne à ses enseignants que les pratiques sociales sont le plus souvent «habillées » (Ubaldi, Coston, Coltice \& 
Philippon, 2006), en intégrant notamment des savoirs d'accompagnement et autres rôles sociaux. On peut alors s'interroger sur la référence de ces derniers. Sont-ils l'expression d'une « culture scolaire » spécifique et autonome ou, au contraire, le reflet d'un univers culturel existant essentiellement en dehors de l'école ? D'après Cogérino (2002), les textes définissant en France les savoirs d'accompagnement les situent sur le registre d'une certaine transversalité. Les savoirs d'accompagnement ne concerneraient donc pas une APS en particulier. Plusieurs exemples issus des 410 projets d'évaluation analysés par Cogérino illustrent cette transversalité: échauffement; arbitrage; analyser une prestation, une performance, des stratégies; tenir une fiche, rédiger régulièrement son bilan; travailler en autonomie; fonction de conseiller, d'aide au plan relationnel. En revanche, d'autres exemples font clairement apparaitre une filiation avec les pratiques sociales de référence : assurer en escalade ; entretien des VTT. Il en va de même des exemples que nous avons extraits d'autres études de notre corpus : en lutte, mettre en place des surfaces de travail sécurisées (Baeza et al., 2009); en athlétisme, donner le départ et chronométrer ses condisciples (David \& Amarouche, 2006) ; en danse, adopter un regard de spectateur (Thorel, 2009); dans les activités gymniques (gymnastique, acrogym, cirque), assurer la parade (Vivier \& Dupaux, 2007). Dans ce cas, les savoirs d'accompagnement et les rôles sociaux ne sont pas des créations scolaires, mais résultent du repérage, puis de la mise en forme, à des fins éducatives, de pratiques, de compétences, de rôles faisant partie intégrante de l'univers culturel pris comme référence, conformément à la chaîne de transposition didactique complexifiée par Perrenoud (1998) (voir figure 1). Cette mise en forme par l'institution scolaire des pratiques sociales de référence consiste alors à faire assumer à un même élève différents rôles (pratiquant, observateur, coach, arbitre, juge, spectateur, responsable du matériel...) qui sont habituellement dévolus à des individus distincts dans l'univers culturel de référence.

Ce constat d'une "culture scolaire», néanmoins assujettie à un univers culturel existant en dehors de l'école majoritairement représenté par les pratiques sportives fédérales, auquel nous arrivons à l'issue de cette revue de littérature, est-il généralisable? Force est de constater que plusieurs obstacles viennent contrecarrer sa généralisation, à commencer par le fait que la majorité des données proviennent du système scolaire français. En effet, si l'on fait abstraction des trois méta-analyses de notre corpus, 44 études ont été réalisées en France contre seulement trois en Belgique francophone, trois au Bénin, deux en Tunisie, deux au Québec, une en Angleterre, une aux États-Unis, une en Suisse, une au Chili et une à Malte. Or, l'enseignement de l'EPS en France est connu pour véhiculer majoritairement une logique sportive, ainsi qu'en témoigne le rejet successif par la corporation des classifications de 1993 et de 1999 qui faisaient appel à des logiques motrices et scolaires (Jarnet, 2009 ; Marsault, 2005). En outre, ce qui semble constituer l'exception, tel que la référence à des activités de loisir ou l'importation sans ajustements de pratiques fédérales à l'école, a précisément été relevé dans des pays minorisés dans notre corpus. Il s'agirait de mener davantage d'études en Angleterre et en Belgique par exemple pour savoir si le recours à des activités de loisir telles que l'aquagym est représentatif de l'EPS enseignée dans ces pays. En attendant, il paraît raisonnable de considérer que les résultats de cette revue de littérature en lien avec notre première question de recherche valent essentiellement pour les curricula d'EPS en France. Un deuxième obstacle réside dans le fait que la très grande majorité des données proviennent de l'enseignement secondaire. Seules, deux études de notre corpus ont eu pour cadre l'enseignement primaire, dans des régions où 
l'EPS est dispensée, totalement ou en partie, par des enseignants spécialistes (Québec, Canada et canton de Genève, Suisse). Or, certaines activités semblent être réservées à ce niveau d'enseignement, à l'instar des jeux traditionnels : "dès que les élèves de l'école primaire pénètrent dans le secondaire, la pratique des jeux traditionnels fond comme neige au soleil » (Dugas, 2009, p. 140). Dans ces conditions, il convient de restreindre la portée de nos résultats à l'enseignement secondaire. Enfin, un troisième obstacle tient au fait que, parmi les études de notre corpus, en faisant à nouveau abstraction des trois métaanalyses, celles où les chercheurs ont réellement pu observer sur le terrain les curricula réellement mis en œuvre par les enseignants d'EPS (curricula réels) sont au nombre de 24 seulement. En corollaire, ces études portaient forcément sur des échantillons réduits d'enseignants alors que les études, plus nombreuses, qui ont recueilli le curriculum déclaré (ou d'autres aspects qui n'apportaient pas de réponse à notre première question de recherche mais bien à la seconde qui sera discutée ensuite) portaient quant à elles sur des échantillons plus statistiquement significatifs. On ne peut dès lors pas exclure un décalage potentiel entre les résultats issus de l'investigation des curricula déclarés et ceux qui auraient été obtenus dans les mêmes contextes par une analyse des curricula réels, tout en sachant que cette analyse était le plus souvent impossible à mettre en œuvre compte tenu de la taille des échantillons étudiés.

\section{2. Comment les enseignants d'EPS effectuent-ils ces choix ?}

17 À l'exception de l'étude menée par Lachheb (2008) où les enseignants d'EPS s'apparentaient à des agents de l'institution scolaire (mais les événements récents en Tunisie aident à comprendre cette exception), les autres études de notre corpus concourent plutôt à une vision de l'enseignant d'EPS comme un acteur social (Amblard et al., 2005), disposant d'une relative marge de liberté dans sa tâche d'élaboration du curriculum réel. Le faible impact de la culture macro-structurelle issue des instructions officielles (déterminant "Institution ») qui ressort de cette revue de littérature résulte sans doute de son caractère lointain (Briot, 1999) : « elle est loin des acteurs dans le temps (elle correspond à un pan théorique de leur formation primitive) et loin dans l'espace parce qu'elle correspond à une vision idéale de la pratique EPS peu en rapport avec les conditions réelles que ces mêmes acteurs vivent au quotidien » (p. 80). D'autres influences curriculaires, méso-structurelles, sont davantage valorisées par les enseignants d'EPS, parmi lesquelles l' " effet équipe d'EPS » (e.g., Briot \& Chifflet, 2001; Garnier, 2008) et les structures de formation continue (e.g., Musard et al., 2008, 2009). La hiérarchisation entre ces déterminants, tous de type "Institution", apparaît ici fondée sur une distinction entre ce qui est proche (qui est valorisé) et ce qui est loin des conditions de travail des enseignants d'EPS (qui est dévalorisé). Dans d'autres études, les instructions officielles voyaient leur portée contrariée par les conceptions ou les orientations de valeur des enseignants d'EPS (Araya-Cortez \& Carlier, 2005 ; Pasco \& Ennis, 2009). Dans ce cas, la hiérarchisation repose plutôt sur une distinction entre les déterminants personnels (qui sont valorisés) et les déterminants institutionnels (qui sont dévalorisés).

Dans cette priorité accordée à ce qui est proche et personnel par rapport à ce qui est loin et institutionnel, nous voyons l'expression de stratégies d'acteurs concourant à l'atteinte d'un "critère de satisfaction conjointe d'intérêts divergents" (Pichault, 1993), en l'occurrence ici les stratégies des enseignants d'EPS pour se conformer à leurs conceptions, leurs orientations de valeur tout en s'adaptant aux attentes de 
l'institution scolaire et de leurs collègues. En fonction de leurs ressources (niveau de formation, connaissances...) et des contraintes qui pèsent sur eux (contraintes matérielles, inspection scolaire...), les enseignants d'EPS se distancieraient plus ou moins des instructions officielles. Cette interprétation est soutenue par plusieurs résultats issus de notre corpus: possédant moins de connaissances, les enseignants d'EPS se conforment davantage aux instructions officielles (Hsouna et al., 2007); soucieux de satisfaire aux exigences minimales de l'institution, ils ne lisent que partiellement ou en surface les textes officiels (Roux-Perez, 2004). L'influence directe des élèves (déterminant "Public ») que nous avons relevée dans quelques études de notre corpus soutient également cette interprétation inspirée des travaux menés en sociologie des organisations (Amblard et al., 2005). En effet, elle apparaissait seulement dans des contextes où les enseignants devaient pallier un manque de formation et de connaissances, dans une APSA particulière (Musard et al., 2008, 2009) ou pour l'enseignement de l'EPS en général (Cordoba, 1996).

D'un autre côté, la " liberté » didactique des enseignants d'EPS s'avère déterminée par les caractéristiques sociologiques du public scolaire auquel ils s'adressent, ce que nous avons catégorisé comme une influence indirecte du déterminant «Public ». Les attentes et les aspirations supposées des élèves contraignent ainsi les enseignants d'EPS intervenant en établissements difficiles à restreindre le choix des pratiques sociales de référence et à organiser leur enseignement à partir d'un modèle largement dominé par la logique sportive (Poggi, 2007 ; Poggi-Combaz, 2002a, 2002b, 2002c). Avec Poggi, nous pensons qu'il s'agit là de l'expression de stratégies de survie (Woods, 1997, cité par Poggi, 2007) qui se distinguent des stratégies d'acteurs précédemment mentionnées par le fait qu'elles résultent de contraintes apparemment moins formelles, moins prescriptives, mais paradoxalement plus déterminantes. Enfin, d'autres influences apparaissent plus inconscientes, à l'image de celle exercée par le genre des enseignants d'EPS (déterminant «Personnel ») sur les curricula qu'ils proposaient réellement à leurs élèves (Couchot-Schiex, 2007; Thorel, 2009; Thorel \& David, 2010). C'est précisément le projet de l'approche socio-didactique que de faire œuvre de dévoilement (Poggi et al., 2010), en rendant visibles, dans le cas qui nous occupe ici, les déterminants indirects ou inconscients des pratiques curriculaires des enseignants d'EPS.

L'influence du genre professionnel (à ne pas confondre avec le genre précédemment cité qui désigne la construction psychosociale du sexe) se situe probablement dans le même registre. Formellement, aucune étude de notre corpus ne fait référence à ce concept pourtant essentiel dans le modèle de Goigoux (2007), supposé jouer un rôle d'intercalaire entre le travail prescrit et le travail réel. Tout au plus nous semble-t-il en avoir trouvé l'expression dans trois études, mais cela nous paraît trop peu pour documenter comment, dans la tâche de construction des curricula en EPS, le genre professionnel peut permettre aux enseignants « de filtrer, d'opérationnaliser et réorganiser les éléments des multiples prescriptions»(p. 58). L'absence de référence au genre professionnel dans les études de notre corpus pourrait s'expliquer par l'ancrage de ce concept dans l'analyse du travail en psychologie ergonomique alors que c'est un cadre théorique socio-didactique qui a délimité notre recherche bibliographique. Néanmoins, après avoir effectué une recherche avec les mots clés " éducation physique » et " genre professionnel » sur le portail Cairn.info qui n’a généré que quatre résultats, nous pouvons affirmer qu'il s'agit en fait d'un champ de recherche qui reste encore largement à explorer. 
Contrairement aux résultats en lien avec la première question de recherche, il nous semble que les résultats que nous venons de discuter en relation avec notre seconde question de recherche sont moins affectés par les limites méthodologiques précédemment soulignées (surreprésentation $\mathrm{du}$ contexte français et de l'enseignement secondaire et minorité d'études ayant réellement investigué les curricula réels). En effet, alors que la première question de recherche (quels contenus résultent des choix opérés par les enseignants d'EPS ?) débouche forcément sur des réponses descriptives nécessitant une généralisation statistique (Yin, 2002) sensible à ces limitations, la seconde question de recherche (comment les enseignants d'EPS effectuent-ils ces choix ?) aboutit plutôt à une ébauche, pour le cas particulier de la construction des curricula en EPS, d'une théorie s'appuyant sur le modèle de l'activité de l'enseignant proposé par Goigoux (2007), complété par la modélisation de Briot (1999). En ce sens, la généralisation dont il est question ici n'est plus de nature statistique mais bien analytique (Yin, 2002), moins sensible à ces limitations.

\section{Conclusion et perspectives}

18 La présente revue de littérature se proposait de réaliser une synthèse des études portant sur les activités curriculaires des enseignants d'EPS. Ces études, au nombre de 62 , se référaient à divers cadres théoriques, la majorité d'entre elles s'appuyant sur un cadre didactique, sociologique ou socio-didactique. Globalement, les résultats indiquent que les enseignants d'EPS ont tendance à privilégier leurs propres conceptions (niveaumicro-structurel) et les références curriculaires plus locales (niveau méso-structurel) dans l'élaboration de leurs curricula, tout en étant en partie contraints par des déterminants institutionnels (niveau macro-structurel) et relatifs au public scolaire auquel ils s'adressent. L'interférence du genre professionnel dans ce processus complexe constitue une problématique de recherche très peu explorée en EPS. Il s'agit là d'un axe de recherche qui pourrait être développé à l'avenir, en profitant du rapprochement auquel on assiste aujourd'hui entre des disciplines telles que la didactique, la sociologie et la psychologie ergonomique.

Les curricula qui résultent des choix des enseignants d'EPS, oscillant entre prescription et liberté, apparaissent « s'alimenter à la même source » de la transposition didactique. En effet, la majorité des études recensées convergent vers une vision selon laquelle les contenus en EPS sont très largement référés à un univers culturel existant en dehors de l'école, largement représenté par les APS institutionnellement organisées par les fédérations et associations sportives. En revanche, les curricula en EPS se différencient plus nettement dès lors qu'on affine le grain d'analyse pour s'intéresser à la mise en forme scolaire de leurs pratiques sociales de référence. Trois limites méthodologiques majeures nous ont amenés à relativiser le constat d'une " source unique » des curricula en EPS: la surreprésentation du contexte français, la surreprésentation de l'enseignement secondaire et la relativement faible proportion d'études ayant réellement investigué les curricula réels en EPS, parmi les études de notre corpus. Ces trois limitations constituent autant de perspectives de recherches pour l'avenir. Ainsi, des chercheurs d'autres pays que la France devraient s'intéresser davantage à ce qui est enseigné en EPS dans leurs systèmes scolaires respectifs. Dans le même ordre d'idée, il serait utile de disposer d'analyses comparatives des instructions officielles récentes pour l'enseignement de l'EPS dans différents pays. Par ailleurs, l'enseignement de l'EPS à l'école primaire souffre visiblement d'un manque d'intérêt des chercheurs concernés 
par la problématique des curricula scolaires. En tirant parti de la diversité des politiques nationales en matière d'enseignement primaire, il pourrait par exemple être intéressant de comparer l'enseignement de l'EPS à l'école primaire dispensé par des enseignants généralistes (comme c'est le cas à notre connaissance en France et dans certains cantons suisses) avec celui dispensé par des enseignants spécialistes (comme c'est le cas à notre connaissance en Belgique, au Québec et dans d'autres cantons suisses). Enfin, il manque des données sur ce qui est réellement enseigné en EPS, les études ayant privilégié jusqu'ici, en cohérence avec les méthodologies quantitatives utilisées, le curriculum déclaré comme objet de recherche. Le grain d'analyse pourrait désormais s'affiner. La recherche sur l'intervention en EPS et en sport pourrait ainsi s'intéresser aux savoirs, aux compétences, aux dispositifs qui caractérisent les situations d'apprentissage réellement proposées par les enseignants d'EPS à leurs élèves. Pour terminer, les effets des curricula réellement proposés aux élèves en EPS gagneraient à faire l'objet d'investigations longitudinales, dont on connait malheureusement le coût et la difficulté de mise en œuvre.

\section{BIBLIOGRAPHIE}

Adé, D., Sève, C., \& Serres, G. (2007). Familiarité avec la discipline sportive enseignée et forme d'activité chez un enseignant stagiaire d'éducation physique : une étude exploratoire. Staps, 76, 51-66.

Amade-Escot, C. (2000). The contribution of two research programs on teaching content: "Pedagogical Content Knowledge" and "Didactics of Physical Education". Journal of Teaching in Physical Education, 20(1), 78-101.

Araya-Cortez, E., \& Carlier, G. (2005). L'éducation physique chilienne entre la tradition et le changement. Staps, 69, 119-134.

Attard, K., \& Armour, K.M. (2005). Learning to become a learning professional: Reflections on one year of teaching. European Journal of Teacher Education, 28(2), 195-207.

Aubert, J., \& Abdi, G. (2002). Représentations sociales de la gymnastique chez des enseignants stagiaires d'éducation physique et sportive et choix d'enseignement. Staps, 59, 9-22.

Auvray, E. (2009). L'enseignement de la natation en EPS entre 1945 et 1995 (second degré), sportivisation et scolarisation : mythe ou réalité ? Staps, 86, 59-77.

Baeza, N., \& Nourrit-Lucas, D. (2009). La « zone de rencontre » en Éducation Physique et Sportive : un espace de construction identitaire. Science \& Motricité, 68, 51-71.

Baeza, N., Nourrit-Lucas, D., \& Bouthier, D. (2009). La « zone de rencontre » en Éducation Physique et Sportive : lieu d'interaction, de construction et de transmission des savoirs. eJRIEPS, 17, 17-36.

Brau-Antony, S. (2001). Les conceptions des enseignants d'éducation physique et sportive sur l'enseignement et l'évaluation des jeux sportifs collectifs : résultats d'une enquête. Staps, 56, 93-108. 
Brière-Guenoun, F. (2008). L'identification des savoirs mobilisés par le professeur d'EPS en situation : le cas du franchissement par redressement au saut de cheval. eJRIEPS, 15, 60-79.

Briot, M., \& Chifflet, P. (2001). Action organisée et choix des contenus d'enseignement en éducation physique et sportive. Application aux objectifs de socialisation et de formation à la citoyenneté. Staps, 55, 101-114.

Buznic, Bourgeacq, P., Terrisse, A., \& Lestel, G. (2008). Expérience personnelle et expérience professionnelle dans l'enseignement de l'EPS : deux études de cas contrastées en didactique clinique. Éducation et Didactique, 2(3), 77-95.

Buznic-Bourgeacq, P., Terrisse, A., \& Margnes, E. (2007). L'expérience personnelle du professeur d'EPS, approche clinique et implications didactiques : une étude de cas d'une enseignante débutante. eJRIEPS, 11, 20-38.

Buznic-Bourgeacq, P., Terrisse, A., Margnes, E. (2010). La transmission du savoir expérientiel en EPS : études de cas et analyses comparatives en didactique clinique. eJRIEPS, 20, 26-47.

Carnus, M.-F., \& Terrisse, A. (2006). La compétence à évaluer : une compétence révélatrice de la professionnalité des enseignants d'EPS. Étude de cas et perspectives pour la formation. Savoirs, 12, 56-74.

Cogérino, G. (2002). Les difficultés de l'évaluation en EP : le cas des savoirs d'accompagnement. Staps, 59, 23-42.

Cogérino, G. (2007). Propos d'enseignants d'éducation physique face à la mixité. Staps, 75, 25-42.

Cogérino, G., Marzin, P., \& Méchin, N. (1998). Prévention santé : pratiques et représentations chez les enseignants d'Éducation Physique et Sportive et de Sciences de la Vie et de la Terre. Recherche et Formation, 28, 9-28.

Combaz, G., \& Hoibian, O. (2008). Le rôle de l'école dans la construction des inégalités de sexe. L'exemple de l'éducation physique et sportive. Travail, genre et sociétés, 20, 129-150.

Combaz, G., \& Hoibian, O. (2009). La légitimité de la culture scolaire mise à l'épreuve. L'exemple de l'éducation physique et sportive dans le second degré en France. Éducation et Sociétés, 23, 189-206.

Cordoba, A. (1996). Regard sur la construction du curriculum réel en éducation physique à l'école primaire. Bildungsforschung und Bildungspraxis, 18(1), 34-51.

Couchot-Schiex, S. (2007). L'univers de l'enseignant est-il genré ? eJRIEPS, 11, 39-55.

Couchot-Schiex, S., Cogérino, G., \& Coltice, M. (2009). Professeurs stagiaires face à l'enseignement en mixité. Carrefours de l'éducation, 27, 169-182.

David, B., \& Amarouche, P. (2006). Approche clinique de l'activité évaluative de professeurs stagiaires d'éducation physique et sportive. eJRIEPS, 9, 4-17.

Debars, C., \& Amade-Escot, C. (2006). Enseigner le badminton à une classe de primo arrivants : incidence du contexte ZEP (zone d'éducation prioritaire) sur la pratique d'intervention. eJRIEPS, 9, 35-43.

Dugas, E. (2009). L'influence d'un stage de formation continue en EPS : un autre regard sur les jeux traditionnels. Savoirs, 20, 134-157.

Frédéric, O., Gribomont, J., \& Cloes, M. (2009). Comparaison des stratégies d'enseignement du basket-ball en milieu scolaire et en milieu sportif. eJRIEPS, 16, 6-21. 
Ferez, S. (2004). De l'expression corporelle aux Activités Physiques Artistiques (A.P.A.) : subversion sexuée et différenciation sexuée en Éducation Physique et Sportive (E.P.S.). Staps, 66, 113-128.

Garnier, A. (2008). Le rapport au savoir de l'enseignant, révélateur du fonctionnement $\mathrm{du}$ système didactique. eJRIEPS, 14, 22-40.

Garnier, A., \& Amade-Escot, C. (2003). L'enseignement de la gymnastique au collège : tensions et injonctions contradictoires à l'origine du rapport au savoir de l'enseignant. eJRIEPS, 4, 23-37.

Genet-Volet, Y., \& Desrosiers, P. (1995). Programmes d'apprentissage sollicitant des actions de coopération-opposition et transposition didactique. Staps, 36, 29-44.

Green, K., Smith, A., \& Thurston, M. (2009). Busy doing nothing? Physical education teachers' perceptions of young people's participation in leisure-sport. Sport, Education and Society, 14(4), 401-420.

Heuser, F. (2008). Du savoir réellement enseigné en EPS au savoir de référence de l'enseignant : étude didactique clinique en karaté. eJRIEPS, 15, 99-112.

Hsouna, H.B., Terrisse, A., \& Berhaim, A. (2007). Le poids de l'expertise dans l'enseignement de l'EPS : signature de l'enseignant et différenciation de pratiques - études de cas. eJRIEPS, 12, 82-98.

Kossivi, A. (2009a). L'analyse comparée des savoirs enseignés en natation dans les classes de 6ème des collèges et lycées du Bénin et de la France. eJRIEPS, 18, 41-67.

Kossivi, A. (2009b). Les contenus du nouveau programme d'EPS définis en termes de compétences au Bénin : une problématique de l'intervention dans l'enseignement secondaire. Staps, 86, 93-108.

Kossivi, A., Minakpon Albert, T., Souaïbou, G., Anicet, A., \& Refuggi, R. (2006). Problématique didactique de l'intégration d'une activité physique spécifique dans l'enseignement de l'éducation physique et sportive aux lycées et collèges du Bénin : le cas du judo. eJRIEPS, 10, 4-15.

Lachheb, M. (2008). L'idéaltype du corps dans l'enseignement de l'éducation physique tunisienne contemporaine. Éducation et Sociétés, 22, 145-159.

Loizon, D., Margnes, E., \& Terrisse, A. (2008). Analyse des pratiques d'enseignement du judo en EPS. eJRIEPS, 14, 63-82.

Maquaire, P. (2007). La place des étirements dans l'échauffement en EPS. Ambivalence et controverse dans une approche préventive des blessures. Staps, 76, 31-49.

Marsault, C. (2005). Les programmations en EPS : la mise en forme des APS. Staps, 67, 9-22.

Mees, V., Carlier, G., \& Renard, J.-P. (2003). L'influence du chef d'établissement sur l'éducation physique et ses déterminants. Staps, 62, 7-19.

Mons, G. (1992). Les représentations du corps expressif en éducation physique et sportive. Revue Française de Pédagogie, 98, 21-27.

Motter, P., Maraite, A.-A., \& Cloes, M. (2007). Solutions imaginées par les enseignantes en éducation physique du secondaire pour lutter contre le phénomène de la dispense dans le cours de natation. eJRIEPS, 11, 106-119.

Musard, M., Robin, J.-F., \& Caty, D. (2009). La construction des cycles d'acrosport : une démarche originale des enseignants d'EPS. eJRIEPS, 16, 73-85.

Musard, M., Robin, J.-F., Nachon, M., \& Gréhaigne, J.-F. (2008). La « composition sous influences » des curricula : un exemple en acrosport. Science \& Motricité, 64, 61-67. 
Pasco, D., \& Ennis, C.D. (2009). Les orientations de valeur des enseignants. Une revue de question en éducation physique. Science \& Motricité, 66, 85-112.

Pasco, D., Kermarrec, G., \& Guinard, J.-Y. (2008). Les orientations de valeur des enseignants d'éducation physique. Influence du sexe, de l'âge et de l'ancienneté. Staps, 81, 89-105.

Poggi, M.-P. (2007). Le modèle sportif : une stratégie de recours en établissement défavorisé. Science \& Motricité, 61, 101-129.

Poggi-Combaz, M.-P. (2002a). L'illusion d'une éducation corporelle commune en éducation physique et sportive. L'année sociologique, 52, 479-505.

Poggi-Combaz, M.-P. (2002b). La construction des inégalités sociales à l'école : l'EPS ne semble pas épargnée. eJRIEPS, 2, 55-68.

Roux-Perez, T. (2004). L'identité professionnelle des enseignants d'EPS : entre valeurs partagées et interprétations singulières. Staps, 63, 75-88.

Roux-Perez, T. (2005). Dynamiques identitaires à l'échelle du temps : une étude de cas chez les enseignants d'éducation physique et sportive. Science \& Motricité, 56, 75-96.

Rovegno, I. (1994). Teaching with a curricular zone of safety: school culture and the situated nature of student teachers' pedagogical content knowledge. Research Quarterly for Exercise and Sport, 65(3), 269-279.

Thorel, S. (2009). Impact de l'univers de danse pris en référence par l'enseignant-e d'EPS sur la coéducation des élèves en danse contemporaine. eJRIEPS, 17, 37-54.

Thorel, S., \& David, B. (2005). La mixité en éducation physique et sportive : pluralité des approches. eJRIEPS, 8, 103-121.

Thorel, S., \& David, B. (2010). Enseignement de la danse contemporaine au collège : genre et coéducation. Carrefours de l'éducation, 29, 39-57.

Turcotte, S., Otis, J., \& Gaudreau, L. (2007). Les objets d'enseignement-apprentissage : éléments d'illustration de l'inclusion de l'éducation à la santé en éducation physique. Staps, 75, 115-129.

Vandevelde, M., \& Amade-Escot, C. (2003). Étude des modalités d'incorporation de savoirs d'experts dans le cadre de la formation continue en EPS. eJRIEPS, 3, 51-66.

Verscheure, I., \& Amade-Escot, C. (2004). Dynamiques différentielles des interactions didactiques selon le genre en EPS. Le cas de l'attaque en volley-ball en seconde. Staps, 66, 79-97.

Vigneron, C. (2006). Les inégalités de réussite en EPS entre filles et garçons : déterminisme biologique ou fabrication scolaire? Revue Française de Pédagogie, 154, 111-124.

Vivier, C., \& Dupaux, J.-J. (2007). Gérer le danger et le risque en EPS. La sécurité des élèves depuis le milieu du XXe siècle : le cas du lycée Victor-Hugo de Besançon. Carrefours de l'éducation, 23, 137-151.

\section{Autres références}

Amblard, H., Bernoux, P., Herreros, G., \& Livian, Y.F. (2005). Les nouvelles approches sociologiques des organisations ( $3^{\text {ème }}$ édition). Paris : Seuil.

Bajoit, G. (2010). Socio-analyse des raisons d'agir. Études sur la liberté du sujet et de l'acteur. Québec : Presses de l'Université Laval.

Briot, M. (1999). Les stratégies des enseignants d'EPS dans le choix de leurs contenus d'enseignement. Revue Française de Pédagogie, 129, 73-85. 
Caillot, M. (1996). La théorie de la transposition didactique est-elle transposable? In, C. Raisky \& M. Caillot (Eds.), Au-delà des didactiques, le didactique. Débats autour de concepts fédérateurs (pp. 19-35). Bruxelles : De Boeck.

Chervel, A. (1988). L'histoire des disciplines scolaires. Réflexions sur un domaine de recherche. Histoire de l'éducation, 38, 59-119.

Chevallard, Y. (1985). La transposition didactique. Du savoir savant au savoir enseigné. Grenoble : La pensée sauvage.

Cloes, M., Lenzen, B., \& Trudel, P. (2009). Analyse de la littérature francophone portant sur l'intervention de l'entraîneur sportif, publiée entre 1988 et 2007. Staps, 83, 7-23.

Clot, Y., \& Faïta, D. (2000). Genres et styles en analyse du travail. Concepts et méthodes. Travailler, $4,7-42$.

Crahay, M. (2006). Dangers, incertitudes et incomplétude de la logique de la compétence en éducation. Revue Française de Pédagogie, 154, 97-110.

Delignières, D., \& Garsault, C. (1999). Connaissances et compétences en EPS. Revue EPS, 280, 43-47. Forquin, J.-C. (1983). La « nouvelle sociologie de l'éducation » en Grande-Bretagne : orientations, apports théoriques, évolution. Revue Française de Pédagogie, 63, 61-79.

Forquin, J.-C. (2008). Sociologie du curriculum. Rennes : PUR.

Goigoux, R. (2007). Un modèle d'analyse de l'activité des enseignants. Education \& Didactique, 1(3), 47-70.

Jarnet, L. (2009). Controverse sur la classification des Activités Physiques et Sportives dans les réformes nationales du collège en France. Staps, 84, 27-40.

Johsua, S. (1996). Le concept de transposition didactique n'est-il propre qu'aux mathématiques? In, C. Raisky \& M. Caillot (Eds.), Au-delà des didactiques, le didactique. Débats autour de concepts fédérateurs (pp. 61-73). Bruxelles : De Boeck.

Johsua, S., \& Lahire, B. (1999). Pour une didactique sociologique. Education et Société, 4, 29-56.

Lahire, B. (2007). La sociologie, la didactique et leurs domaines scientifiques. Education \& Didactique, 1(1), 73-81.

Martinand, J.-L. (1981). Pratiques sociales de référence et compétences techniques. À propos d'un projet d'initiation aux techniques de fabrication mécanique en classe de quatrième. In, A. Giordan (Ed.), Diffusion et appropriation du savoir scientifique : enseignement et vulgarisation. Actes des Troisièmes Journées Internationales sur l'Éducation Scientifique (pp. 149-154). Paris : Université Paris 7.

Martinand, J.-L. (2001). Pratiques de référence et problématique de la référence curriculaire. In A. Terrisse (Ed.), Didactique des disciplines. Les références au savoir (pp. 17-24). Bruxelles : De Boeck Université.

Musard, M., Mahut, N., \& Robin, J.-F. (2002). Quel processus de construction des activités scolaires en EPS ? eJRIEPS, 2, 43-54.

Perrenoud, P. (1998). La transposition didactique à partir des pratiques : des savoirs aux compétences. Revue des sciences de l'éducation, 24(3), 487-514.

Pichault, F. (1993). Ressources humaines et changement stratégique. Vers un management politique. Bruxelles : De Boeck Université. 
Poggi, M.-P, Verscheure, I., Musard, M., \& Lenzen, B. (2010). Vers une approche socio-didactique en intervention. In M. Musard, M. Loquet \& G. Carlier (Eds.), Sciences de l'intervention en EPS et en sport (pp. 159-178). Paris : Éditions EP\&S.

Ubaldi, J.-L., Coston, A., Coltice, M., \& Philippon, S. (2006). Cibler, habiller, intervenir pour permettre à nos élèves d'apprendre en EPS. Les cahiers du C.E.D.R.E., 6, 7-31.

Verret, M. (1975). Le temps des études. Paris : Champion.

Yin, R.K. (2002). Case study research. Design and methods (3rd edition). Newbury Park, CA: Sage.

\section{RÉSUMÉS}

Ce texte se propose de passer en revue les connaissances empiriques sur le rôle des enseignants d'éducation physique et sportive (EPS) dans l'élaboration des curricula et sur les curricula en EPS qui en résultent. S'appuyant sur un modèle d'analyse existant de l'activité de l'enseignant et sur une recension des principaux travaux conduits ces vingt dernières années sur la problématique étudiée (corpus de 62 recherches), cette revue de littérature confirme l'influence de déterminants institutionnels, personnels et relatifs au public scolaire sur les pratiques curriculaires des enseignants d'EPS. Les curricula qui en résultent apparaissent « s'alimenter à la même source " si on considère leur référence quasi unanime à un univers culturel existant en dehors de l'école, largement représenté par les APS institutionnellement organisées par les fédérations et associations sportives. En revanche, ils se différencient plus nettement lorsqu'on prend en compte la mise en forme scolaire de leurs pratiques sociales de référence. La surreprésentation du contexte français, de l'enseignement secondaire et des études ayant investigué le curriculum déclaré et non le curriculum réellement mis en œuvre par les enseignants d'EPS limite toutefois la portée des résultats de cette revue de littérature.

This paper reviews the literature on the role of physical education (PE) teachers in curriculum construction, and on what is taught in PE. It relies on a model of teacher activity and a recension of 62 studies made on the subject. This literature review confirms the influence of institutional and personal determinants as well as determinants relative to school environment on PE teachers' curricular practices. What is taught in PE mainly refers to sport activities, but these are more or less modified and adapted for school purposes. French studies, studies conducted at secondary school and studies of discourses rather than practices are overrepresented. That limits the significance of the results of this literature review.

\section{INDEX}

Mots-clés : curriculum, sociologie, didactique, éducation physique, enseignant, référence

\section{AUTEUR \\ BENOÎT LENZEN}

Université de Genève, Suisse 